Pacific Journal of Mathematics

UNIFORM APPROXIMATION BY POLYNOMIALS WITH 


\title{
UNIFORM APPROXIMATION BY POLYNOMIALS WITH INTEGRAL COEFFICIENTS II
}

\author{
Le Baron O. Ferguson
}

Let $A$ be a discrete subring of $C$ of rank 2. Let $X$ be a compact subset of $C$ with transfinite diameter not less than unity or with transfinite diameter less than unity, void interior, and connected complement. In an earlier paper we characterized the complex valued functions on $X$ which can be uniformly approximated by elements from the ring of polynomials $A[z]$. In this paper the same problem is studied where $X$ is a compact subset of $C$ with transfinite diameter $d(X)$ less than unity and with nonvoid interior. It is also studied for certain compact subsets of $C^{n}$ where $n$ is any positive integer. These subsets will have the property that every continuous function holomorphic on the interior is uniformly approximable by complex polynomials. A large class of sets of this type is shown to exist.

We endeavor to follow the notation and terminology of Bourbaki [2]. Throughout, $X$ is a compact subset of $C^{n}, n \geqq 1$. We use the symbol $z$ to stand for the $n$-tuple of complex numbers $\left(z_{1}, \cdots, z_{n}\right) \in C^{n}$. If $R$ is any subring of $C, R[z]$ will denote $R\left[z_{1}, \cdots, z_{n}\right]$, the ring of polynomials in $z_{1}, \cdots, z_{n}$ with coefficients in $R$. When $z$ is an element of $C^{n}$, we define $|z|$ by

$$
|z|=\left(\left|z_{1}\right|^{2}+\cdots+\left|z_{n}\right|^{2}\right)^{1 / 2} \geqq 0 \text {. }
$$

We write

$$
\sum_{k \in N^{n}} a_{k} z^{k}
$$

to denote

$$
\sum_{k_{1}, \cdots, k_{n}=0}^{\infty} a_{k_{1}, k_{2}, \cdots, k_{n}} z_{1}^{k_{1}} z_{2}^{k_{2}} \cdots z_{n}^{k_{n}}
$$

(Here $N$ stands for the nonnegative integers.)

If $f$ is a complex valued function on $X$ and $R$ a subring of $C$, we say that $f$ is $R$-approximable on $X$ if for any $\varepsilon>0$ there exists $p$ in $R[z]$ such that

$$
\|f-p\|_{X}=\sup _{z \in X}|f(z)-p(z)|<\varepsilon .
$$

2. Polynomial convexity. If $X$ is a compact subset of $C^{n}$, we define its polynomial hull $h(X)$ by 


$$
h(x)=\left\{z \in C^{n}:|p(z)| \leqq\|p\|_{X} \text { for all } p \in C[z]\right\} .
$$

It is easy to see that $h(X)$ is also compact. If $X=h(X)$ we say that $X$ is polynomially convex. It suffices to consider only such $X$ by the following (Wermer [7, p. 53]).

Proposition 2.1. Let $f$ be a complex valued function on $X$ and $P$ any subset of $C[z]$. If $f$ is uniformly approximable by elements of $P$, then there exists exactly one extension $f^{\prime}$ of $f$ to $h(X)$ such that $f^{\prime}$ is uniformly approximable by elements of $P$.

If $X$ is a subset of $C$ and $t_{m}(z, X)$ is the Chebyshev polynomial of degree $m$ associated with $X$ (Ferguson [3, §3]) then $t_{m}(z, X)$ is the same as $t_{m}(z, h(X))$ which immediately gives $d(X)=d(h(X))$, where $d$ denotes transfinite diameter. In this case $(n=1)$ we also have that $h(X)$ equals $X$ union the bounded components of its complement (Hormander [5, p. 8, Th. 1.3.3]).

By utilizing the maximal principle in each variable separately one can verify the following examples which will be needed later.

ExAmple 2.2. If $X$ is defined by

$$
X=\left\{z \in C^{n}:\left|z_{1}\right|=\left|z_{2}\right|=\cdots=\left|z_{n}\right|=1\right\}
$$

then we have

$$
h(X)=\left\{z \in C^{n}:\left|z_{i}\right| \leqq 1,1 \leqq i \leqq n\right\} .
$$

This example brings out two differences between the cases $n=1$ and $n>1$. First, it is clear that $X$ does not contain the topological boundary of $h(X)$ in this example if $n>1$, although it does if $n=1$. Secondly, we have seen that for $n=1$, if $C \sim X$ (complement of $X$ in $C$ ) is connected, then $X=h(X)$. This is also false for $n>1$.

EXAMPLE 2.3. If $n>1$ and

$$
X=\left\{z \in C^{n}:\left|z_{1}\right|=1, z_{2}=z_{3}=\cdots=z_{n}=0\right\},
$$

then

$$
h(X)=\left\{z \in C^{n}:\left|z_{1}\right| \leqq 1, z_{2}=z_{3}=\cdots=z_{n}=0\right\}
$$

3. Necessary conditions. Throughout this section let $R$ be any discrete subring of $\boldsymbol{C}$.

Proposition 3.1. If $f$ is a complex valued function on a compact subset $X$ of $C^{n}$ and $f$ is $R$-approximable on $X$, then $f$ has a unique extension $f^{\prime}$ to $h(X)$ which is continuous on $h(X)$, holomorphic on 
$h(X)^{\circ}$ (where superscript ${ }^{\circ}$ denotes “interior"), and $R$-approximable on $h(X)$.

Proof. The proof is immediate from 2.1 since uniform limits preserve continuity and holomorphicity.

An example of the use of 3.1 is the following. Let

$$
X=\left\{z \in C: \delta_{1} \leqq|z| \leqq \delta_{2}\right\},
$$

where $0<\delta_{1}<\delta_{2}<1$, and let $f$ be the function $z+1 / 2$ restricted to $X$. By $\S 2, h(X)=\delta_{2} D$ where $D$ is the closed unit disk in $C$. By the principle of holomorphic continuation, the only continuous extension of $f$ to $h(X)$ which is holomorphic on $\delta_{2} D^{\circ}$ is the function $z+1 / 2$ restricted to $\delta_{2} D$. But this function is not $R$-approximable on $\delta_{2} D$ for any discrete ring $R$ since any polynomial $p \in R[z]$ has as its value at $z=0$ an element of $R$ and every nonzero element of $R$ has modulus at least unity. Thus $f$ is not $R$-approximable on $X$ for any discrete ring $R$.

In the case $X \subset C$ it is easy to use 3.1 as follows. Given a complex valued function $f$ on $X$ we seek an extension $f^{\prime \prime}$ of $f$ to $h(X)$ which is continuous on $h(X)$ and holomorphic on $h(X)^{\circ}$. If none such exists, then $f$ is not $R$-approximable on $X$. If such an $f^{\prime \prime}$ exists, then $f$ is $R$-approximable on $X$ if and only if $f^{\prime \prime}$ is $R$-approximable on $h(X)$. This is true since if $f$ is $R$-approximable on $X$, then by 3.1 there exists an $R$-approximable extension $f^{\prime}$ of $f$ to $h(X)$ which is continuous on $h(X)$ and holomorphic on $h(X)^{\circ}$. By $\S 2$ and the maximum modulus principle $f^{\prime}$ is then uniquely determined by $f$, and so $f^{\prime}=f^{\prime \prime}$ which shows that $f^{\prime \prime}$ is $R$-approximable. The converse is obvious.

This procedure is contingent upon the fact that if $X \subset C$, then there is at most one extension $f^{\prime}$ of $f$ to $h(X)$ which is continuous on $h(X)$ and holomorphic on $h(X)^{\circ}$. If $X \subset C^{n}, n>1$, the extension need not be unique, as the following example shows.

Let

$$
X=\left\{z:\left|z_{1}\right|=1, z_{2}=\cdots=z_{n}=0\right\} .
$$

We know from 2.3 that

$$
h(X)=\left\{z:\left|z_{1}\right| \leqq 1, z_{2}=\cdots=z_{n}=0\right\} .
$$

If we define $f(z)=1$ for all $z$ in $X$, then $f$ is certainly $Z$-approximable on $X$. Also, the function

$$
f^{\prime \prime}(z)=\left|z_{1}\right| \quad z \in h(X)
$$


is an extension of $f$ to $h(X)$ which is continuous on $h(X)$ and holomorphic on $h(X)^{\circ}$ (indeed, $h(X)^{\circ}$ is empty). But it is clear that the unique extension $f^{\prime}$ of $f$ which is $Z$-approximable on $h(X)$ is simply $f^{\prime}(z)=1, z \in h(X)$.

Proposition 3.2. Let $X$ be a compact subset of $C^{n}$ and $f$ a map from $X$ into $C$. Then if $f$ is $R$-approximable on $X$, it has a power series expansion about each point in $R^{n} \cap X^{\circ}$ with coefficients $R$.in

Proof. Let $z_{0}$ be an element of $R^{n} \cap X^{\circ}$. If $\left(p_{n}\right)$ is a sequence of polynomials in $R[z]$ which tends to $f$ uniformly on $X$ then by Bochner and Martin [1, p. 23] the coefficients of the power series expansions of the $p_{n}$ about $z_{0}$ converge to the respective coefficients of the power series expansion of $f$ about $z_{0}$. Since $R$ is closed in $C$ we are done.

For completeness we quote Ferguson [3, Prop. 3.1].

Proposition 3.3. Let $X$ be a compact subset of $C$ with $d(X) \geqq 1$. Then a complex valued function $f$ on $X$ is $R$-approximable on $X$ if and only if it is already an element of $R[z]$.

By restricting a complex valued function on $C^{n}$ to a function of one complex variable, in certain ways, we can get additional necessary conditions for approximability as follows. For $a \in R^{n-1}$ put

$$
X_{k}=\left\{z \in C:\left(a_{1}, \cdots, a_{k-1}, z, a_{k}, \cdots, a_{n-1}\right) \in X\right\}
$$

and $g(z)=f\left(a_{1}, \cdots, a_{k-1}, z, a_{k}, \cdots, a_{n-1}\right) . \quad X_{k}$ is a compact subset of $C$ and if $f$ is $R$-approximable on $X$, then clearly $g$ is $R$-approximable on $X_{k}$.

4. Sufficient conditions. Throughout this section we take $A$ to be a discrete subring of $C$ with rank 2 . We also always take $X$ to be polynomially convex in view of $\S 2$. The following is obvious.

Proposition 4.1. If $z^{\prime}$ is an element of $A^{n}$, then a complex valued function $f(z)$ is $A$-approximable on a subset $X$ of $C^{n}$ if and only if $f\left(z-z^{\prime}\right)$ is $A$-approximable on $z^{\prime}+X$.

The following definition partially delineates the class of sets to which the main theorem of this section applies.

Definition 4.2. A compact subset $X$ on $C^{n}$ is said to be Mergelyan if it satisfies the following condition. Any complex valued function which is continuous on $X$ and holomorphic on $X^{\circ}$ is $C$-approximable on $X$. 
This terminology is motivated by the following theorem, first proved in Mergelyan [6].

THEOREM 4.3. A compact subset $X$ of $C$ is Mergelyan if and only if its complement $C \sim X$ is connected.

By $\S 2$ we see that another equivalent condition is that $X$ be polynomially convex, at least in case $X \subset C$. The answer to the corresponding question for compact subsets of $C^{n}, n>1$, is not known to the writer, but is certainly more complicated. Indeed, we can see that Lavrent'ev's theorem (Ferguson [3, Prop. 5.1]), which is a consequence of Mergelyan's theorem, fails if we attempt to apply it word for word to compact subsets of $C^{n}, n>1$, as follows. Let $X$ be as in 2.3. Then $h(X)$ has void interior, since $n>1$, and its complement $C^{n} \sim h(X)$ is connected as can be seen by elementary means. Thus $h(X)$ satisfies the hypotheses of Mergelyan's theorem except that $X \not \subset C$. The continuous function $f^{\prime \prime}(z)=\left|z_{1}\right|, z \in h(X)$, is not $C$-approximable on $h(X)$ since by 2.1 there exists exactly one such approximable extension of $f(z)=1, z \in X$, to $h(X)$ and this is obviously the function $f^{\prime}(z)=1, z \in h(X)$. Thus neither Lavrent'ev's nor Mergelyan's theorem extend to higher dimensions.

A theorem which does extend, in a sense, is Runge's theorem. In one of its forms this theorem states that every complex valued function holomorphic in a neighborhood of a compact set $X$ of $C$ is $C$-approximable on $X$ if and only if $C \sim X$ is connected. From $\S 2$ we know that $C \sim X$ is connected if and only if $X$ is polynomially convex. It is this last criterion which remains in force as we pass to higher dimensions.

Proposition 4.4. Let $X$ be a polynomially convex subset of $C^{n}$. Then any function which is holomorphic in a neighborhood of $X$ is $C$-approximable on $X$.

Proof. This is a consequence of Hormander [5, p. 91, 4.3.2].

The following exhibits a large class of Mergelyan subsets of $C^{n}$, for any $n$.

Proposition 4.5. Let $X$ be a polynomially convex subset of $C^{n}$ such that there exists $z^{\prime} \in X$ and $\delta>0$ such that whenever $1<a<$ $1+\delta$ we have

$$
\left(a X-(a-1) z^{\prime}\right)^{\circ} \supset X .
$$

That is, the dilation of $X$ about $z^{\prime}$ of magnitude $a$ is a neighborhood 
of $X$ for all $a \in(1,1+\delta)$. Then $X$ is Mergelyan.

Proof. Choose $\varepsilon>0$ and without loss of generality assume $z^{\prime}=0$. For $1<a<1+\delta$ put $f_{a}(z)=f(z / a) ; f_{a}$ is holomorphic in $a X$, and by 4.4 there exists $p_{a} \in C[z]$ such that $\left\|f_{a}-p_{a}\right\|_{X}<\varepsilon / 2$. It is clear that $\left\|f_{a}-f\right\|_{X} \rightarrow 0$ as $a \rightarrow 1$. Hence if $a$ is chosen so that $\left\|f_{a}-f\right\|_{X}<\varepsilon / 2$, then $\left\|p_{a}-f\right\|_{X}<\varepsilon$.

As an example of this consider

$$
X=\left\{z \in C^{n}:\left|z_{1}\right| \leqq 1, \cdots,\left|z_{n}\right| \leqq 1\right\} .
$$

We know from $\S 2$ that $X$ is polynomially convex. It is clear that the remaining hypothesis of 4.5 is satisfied with $z^{\prime}=0$ and $\delta=\infty$, hence $X$ is Mergelyan. Clearly, the same argument applies for any closed polydisk.

The following result follows from definitions by considering the functions $f(z)=e^{a z}$ for all complex numbers $a$.

Proposition 4.6. If $X$ is a compact convex subset of $C^{n}$, then $X$ is polynomially convex.

EXAMPLE 4.7. Let

$$
X=\left\{z \in C^{n}:|z| \leqq 1\right\} .
$$

Clearly $X$ is compact and convex $\left(\left|z+z^{\prime}\right| \leqq|z|+\left|z^{\prime}\right|\right)$ and so by 4.6 $X$ is polynominally convex. Clearly, for any $a>1$

$$
(a X)^{\circ}=\left\{z \in C^{n}:|z|<a\right\} \supset X .
$$

Thus, by $4.5, X$ is Mergelyan.

It is clear that any translation or nonzero dilation of a Mergelyan set is Mergelyan.

The following is the main result of the paper.

THEOREM 4.8. Let $X$ be a Mergelyan subset of the open unit polycylinder with $0 \in X^{\circ}$. If $f$ is any complex valued function on $X$, the following are equivalent:

(i) The function $f$ is A-approximable on $X$;

(ii) The function $f$ is continuous on $X$, holomorphic on $X^{\circ}$ and the coefficients of its power series expansion about the origin lie in A.

Proof. By 3.1 and 3.2 we have that (i) implies (ii).

Conversely, assume that (ii) holds and $\varepsilon>0$. Let $\rho=\max _{1 \leqq i \leqq n}\left\|z_{i}\right\|_{X}$. 
Since $X$ is compact, $\rho<1$. It is easy to see that

$$
\sum_{k \in N^{n}} \rho^{k_{1}+\cdots+k_{n}}<\infty \text {. }
$$

Let $\delta>0$ be such that for any $z \in C$ there is an $a \in A$ such that $|z-a|<\delta$. The existence of such a $\delta$ is easily demonstrated (Ferguson $[3, \S 2])$. There exists a finite subset $F$ of $N^{n}$ such that

$$
\sum_{k \in N^{n} \sim F} \rho^{k_{1}+\cdots+k_{n}}<\frac{\varepsilon}{3 \delta} .
$$

Since $X$ is Mergelyan by hypothesis, there exists a sequence of polynomials $\left(p_{m}\right)$ in $C[z]$ such that $\left(p_{m}\right) \rightarrow f$ uniformly on $X$. Let

$$
p_{m}=\sum_{k \in N^{n}} a_{k}^{(m)} z^{k}
$$

Where, for each $m$, all but finitely many of the $a_{c k}^{(m)}$ are zero, and

$$
f=\sum_{k \in N^{n}} a_{k} z^{k}
$$

in a neighborhood of the origin. Then, as noted in the proof of 3.2 , for each $k \in N^{n}$

$$
a_{k}^{(m)} \rightarrow a_{k} \quad \text { as } \quad m \rightarrow \infty .
$$

Thus there exists a positive integer $N$ such that $m>N$ implies

$$
\left\|p_{m}-f\right\|_{X}<\frac{\varepsilon}{3}
$$

and

$$
\left|a_{k}^{(m)}-a_{k}\right|<\frac{\varepsilon}{3 M(\operatorname{card} F)} \quad \text { for } k \in F,
$$

where $M=\max _{k \in F}\left\|z^{k}\right\|_{X}$. Thus if $m>N$ and $\left[p_{m}\right]$ denotes the polynomial $p_{m}$ with each coefficient replaced by a nearest element of $A$, we have

$$
\begin{aligned}
\left\|p_{m}-\left[p_{m}\right]\right\| & \leqq \sum_{k \in F}\left|a_{k}^{(m)}-a_{k}\right|\left\|z^{k}\right\|+\sum_{k \notin F} \delta\left\|z^{k}\right\| \\
& <\sum_{k \in F} \frac{\varepsilon}{3 M(\operatorname{card} F)} M+\delta \sum_{k \notin F} \rho^{k_{1}+\cdots+k_{n}} \\
& <\frac{\varepsilon}{3}+\frac{\varepsilon}{3}=2 \frac{\varepsilon}{3} .
\end{aligned}
$$

This estimate together with (1) gives

$$
\left\|\left[p_{m}\right]-f\right\|<\varepsilon \text {. }
$$


The problem is, in the sense given by 4.1, invariant under translation by elements of $A^{n}$; and so the theorem could have been stated for Mergelyan subsets of an open unit polydisk centered at any element of $A^{n}$.

The following example is the solution of the problem which motivated this paper.

EXAMPLE 4.9. Let $X$ be a circle of radius $r<1$ and centered at the origin of the complex plane. There is no loss of generality in the assumption $r<1$ since it is well known that $d(X)=r$; and if $d(X) \geqq 1$, the problem is trivial by 3.3. Let $f$ be a continuous complex valued function defined on $X$. By $\S 2, h(X)=r D$, where $D$ is the closed unit disk. By 3.1, in order that $f$ be approximable on $X$ it is necessary that $f$ have a continuous extension to $h(X)$ which is holomorphic on $h(X)^{\circ}$ and $A$-approximable on $h(X)$.

It is well known that a continuous function $g$ defined on $|z|=1$ has an extension which is continuous on $|z| \leqq 1$ and holomorphic on $|z|<1$ if and only if the Fourier coefficients $\hat{g}(n)$ of the function are all zero for $n<0$ (Hoffman [4, p. 42]). It is easy to see from this that $f$ has a continuous extension $f^{\prime}$ to $h(X)$ which is holomorphic on $h(X)^{\circ}$ if and only if

$$
\int_{|z|=1} f(r z) z^{m} d z=0 \text { for } m=0,1, \cdots,
$$

or equivalently,

$$
r^{-(m+1)} \int_{X} f(z) z^{m} d z=0 \text { for } m=0,1, \cdots .
$$

It is clear from the maximum modulus principle that $f^{\prime}$ is uniquely determined, if it exists. Then by 3.2 , if $f$ is to be $A$-approximable on $X$ the coefficients of the power series expansion of $f^{\prime}$ about 0 must be in $A$, that is,

$$
\frac{1}{2 \pi i} \int_{X} f(z) z^{-(m+1)} d z \in A \text { for } m=0,1, \cdots \text {. }
$$

In summary then, in order that $f$ be $A$-approximable on $X$ it is necessary that

$$
\frac{1}{2 \pi i} \int_{X} f(z) z^{m-1} d z\left\{\begin{array}{lll}
=0 & \text { for } & m=1,2, \cdots \\
\in A & \text { for } & m=0,-1, \cdots .
\end{array}\right.
$$

These conditions are also sufficient by the preceding discussion and 4.8. 


\section{REFERENCES}

1. S. Bochner and W. T. Martin, Several complex variables, Princeton University Press, Princeton, N. J., 1948.

2. N. Bourbaki, Eléments de mathématique, Actualités Sci. et Ind., Hermann et Cie., Paris.

3. L. B. O. Ferguson, Uniform approximation by polynomials with integral coefficients $I$, to appear in this Journal.

4. K. Hoffman, Banach spaces of analytic functions, Prentice-Hall Series in Modern Analysis, Prentice-Hall, Inc., Englewood Cliffs, N. J., 1962.

5. L. Hormander, An Introduction to complex analysis in several variables, The University Series in Higher Mathematics, D. Van Nostrand Co., Inc., Princeton, N. J., 1966.

6. S. N. Mergelyan, On the representation of functions by series of polynomials on closed sets, Soviet Math. Dokl. 78 (1951), 405-408.

7. J. Wermer, Banach algebras and analytic functions, Advances in Mathematics 1 (1961), 51-102.

Received November 17, 1966, and in revised form August 16, 1967. This work forms a part of the author's dissertation under the direction of Professor Edwin Hewitt.

UNIVERSITY OF WASHINGTON

UNIVERSITy OF CALIFORNIA, RIVERSIDE 



\title{
PACIFIC JOURNAL OF MATHEMATICS
}

\author{
EDITORS
}

\author{
H. ROYDEN \\ Stanford University \\ Stanford, California
}

\author{
J. P. JANS \\ University of Washington \\ Seattle, Washington 98105
}

\section{J. DUGUNDJI}

Department of Mathematics University of Southern California Los Angeles, California 90007

\section{RICHARD ARENS}

University of California Los Angeles, California 90024

\section{ASSOCIATE EDITORS}

\author{
E. F. BECKENBACH
}

B. H. NEUMANN

F. WoLF

K. YosidA

\section{SUPPORTING INSTITUTIONS}

\author{
UNIVERSITY OF BRITISH COLUMBIA \\ CALIFORNIA INSTITUTE OF TECHNOLOGY \\ UNIVERSITY OF CALIFORNIA \\ MONTANA STATE UNIVERSITY \\ UNIVERSITY OF NEVADA \\ NEW MEXICO STATE UNIVERSITY \\ OREGON STATE UNIVERSITY \\ UNIVERSITY OF OREGON \\ OSAKA UNIVERSITY \\ UNIVERSITY OF SOUTHERN CALIFORNIA
}

\author{
STANFORD UNIVERSITY \\ UNIVERSITY OF TOKYO \\ UNIVERSITY OF UTAH \\ WASHINGTON STATE UNIVERSITY \\ UNIVERSITY OF WASHINGTON \\ AMERICAN MATHEMATICAL SOCIETY \\ CHEVRON RESEARCH CORPORATION \\ TRW SYSTEMS \\ NAVAL WEAPONS CENTER
}

Mathematical papers intended for publication in the Pacific Journal of Mathematics should be in typed form or offset-reproduced, double spaced with large margins. Underline Greek letters in red, German in green, and script in blue. The first paragraph or two must be capable of being used separately as a synopsis of the entire paper. It should not contain references to the bibliography. Manuscripts, in duplicate if possible, may be sent to any one of the four editors. All other communications to the editors should be addressed to the managing editor, Richard Arens, University of California, Los Angeles, California 90024.

Each author of each article receives 50 reprints free of charge; additional copies may be obtained at cost in multiples of 50 .

The Pacific Journal of Mathematics is published monthly. Effective with Volume 16 the price per volume (3 numbers) is $\$ 8.00$; single issues, $\$ 3.00$. Special price for current issues to individual faculty members of supporting institutions and to individual members of the American Mathematical Society: $\$ 4.00$ per volume; single issues $\$ 1.50$. Back numbers are available.

Subscriptions, orders for back numbers, and changes of address should be sent to Pacific Journal of Mathematics, 103 Highland Boulevard, Berkeley 8, California.

Printed at Kokusai Bunken Insatsusha (International Academic Printing Co., Ltd.), 7-17, Fujimi 2-chome, Chiyoda-ku, Tokyo, Japan.

PUBLISHED BY PACIFIC JOURNAL OF MATHEMATICS, A NON-PROFIT CORPORATION

The Supporting Institutions listed above contribute to the cost of publication of this Journal, but they are not owners of publishers and have no responsibility for its content or policies. 


\section{Pacific Journal of Mathematics}

Vol. 26, No. 2 December, 1968

Seymour Bachmuth and Horace Yomishi Mochizuki, Kostrikin's theorem on

Engel groups of prime power exponent ....................

Paul Richard Beesack and Krishna M. Das, Extensions of Opial's inequality ...................................... 215

John H. E. Cohn, Some quartic Diophantine equations .............. 233

H. P. Dikshit, Absolute $(C, 1) \cdot\left(N, p_{n}\right)$ summability of a Fourier series and its conjugate series ............................... 245

Raouf Doss, On measures with small transforms ................. 257

Charles L. Fefferman, $L_{p}$ spaces over finitely additive measures........ 265

Le Baron O. Ferguson, Uniform approximation by polynomials with integral coefficients. II...................................

Takashi Ito and Thomas I. Seidman, Bounded generators of linear

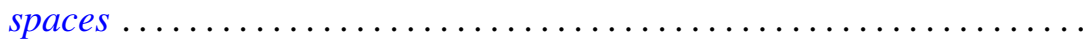

Masako Izumi and Shin-ichi Izumi, Nörlund summability of Fourier series ..........................................

Donald Gordon James, On Witt's theorem for unimodular quadratic

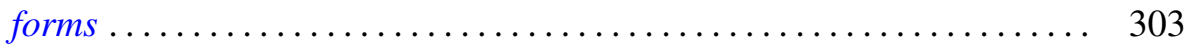

J. L. Kelley and Edwin Spanier, Euler characteristics .............. 317

Carl W. Kohls and Lawrence James Lardy, Some ring extensions with matrix

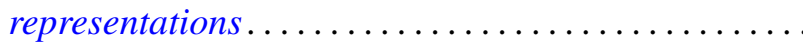

Ray Mines, III, A family of functors defined on generalized primary groups ....

Louise Arakelian Raphael, A characterization of integral operators on the space of Borel measurable functions bounded with respect to a weight function....

Charles Albert Ryavec, The addition of residue classes modulo $n .$.

H. M. (Hari Mohan) Srivastava, Fractional integration and inversion formulae associated with the generalized Whittaker transform ...

Edgar Lee Stout, The second Cousin problem with bounded data ...

Donald Curtis Taylor, A generalized Fatou theorem for Banach

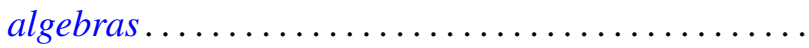

Bui An Ton, Boundary value problems for elliptic convolution equations of Wiener-Hopf type in a bounded region...

Philip C. Tonne, Bounded series and Hausdorff matrices for absolutely convergent sequences... 